\title{
Psychoanalyse und Setting
}

\author{
Ita Grosz-Ganzoni (Zürich)
}

Zusammenfassung: Im Zusammenhang mit den aktuellen Diskussionen und den daraus folgenden Entscheidungen für die Weiterbildung zu PsychotherapeutInnen am PSZ, ist es das Anliegen der Autorin darzulegen, dass die Settingdichte in der psychoanalytischen Arbeit spezifische Folgen für die PatientInnen und AnalytikerInnen/PsychotherapeutInnen hat. Sie plädiert für die Wahrnehmung der Unterschiede zwischen hochfrequenten und niederfrequenten Arbeitsbedingungen, zeigt jedoch auf, dass sich daraus keine Unterschiede bezüglich der psychoanalytischen Selbsterfahrung als Basis für psychotherapeutische Arbeit folgern lassen.

Schlüsselwörter: Purifizierung, das notwendige Gift der Analyse, Schonung des Affektlebens der PsychoanalytikerInnen, hochfrequente Analyse, niederfrequente psychoanalytische Psychotherapie: Besonderheiten bei unterschiedlicher Settingdichte.

\section{Vom Umgang mit gefährlichen Substanzen in der psychoanalytischen Arbeit}

1912 schreibt Freud in seiner Arbeit «Ratschläge für den Arzt bei der psychoanalytischen Behandlung» die legendären Urgesetze der Technik, die er selber als «sehr einfache» bezeichnet, nämlich, sich nichts besonders merken zu wollen und allem, was man zu hören bekomme, die nämliche «gleichschwebende Aufmerksamkeit» entgegenzubringen. Er rät ab vom Versuch, Behandlungsverläufe protokollieren zu wollen, plädiert für ein voraussetzungsloses und unbefangenes Sicheinlassen ohne zu spekulieren, ohne zu grübeln, solange man analysiere, ja sogar erst dann das gewonnene Material der synthetischen Denkarbeit zu unterziehen, nachdem die Analyse abgeschlossen sei. Er greift dann zum viel diskutierten Bild des Chirurgen, der alle seine Affekte beiseite drängt und seine geistigen Kräfte auf das alleinige Ziel konzentriert, die Operation so kunstgerecht wie möglich zu vollziehen. Die empfohlene Gefühlskälte bewirke u.a. auch die wünschenswerte Schonung des Affektlebens des Arztes. In einem späteren Abschnitt verwendet er 
ein weiteres bemerkenswertes Bild dessen, was in der Analyse geschehen muss, damit sie gelingt:

«Er (der Arzt [IG]) soll dem gebenden Unbewussten des Kranken sein eigenes Unbewusstes als empfangendes Organ zuwenden, sich auf den Analysierten einstellen wie der Receiver des Telefons zum Teller eingestellt ist. Wie der Receiver die von Schallwellen angeregten Schwankungen der Leitung wieder in Schallwellen verwandelt, so ist das Unbewusste des Arztes befähigt, aus den ihm mitgeteilten Abkömmlingen des Unbewussten dieses Unbewusste, welches die Einfälle des Kranken determiniert hat, wiederherzustellen» (Freud 1912e: 381-382).

Freud fährt dann fort, dass der Arzt selber eine «psychologische Bedingung in weitem Ausmaße» erfüllen muss, um sich seines Unbewussten als Instrument bei der Analyse zu bedienen. Scharf formuliert er: «Es genügt nicht hiefür, dass er selbst ein annähernd normaler Mensch sei, man darf vielmehr die Forderung aufstellen, dass er sich einer psychoanalytischen Purifizierung unterzogen und von jenen Eigenkomplexen Kenntnis genommen habe, die geeignet wären, ihn in der Erfassung des vom Analysierten Dargebotenen zu stören» (Freud 1912e: 382).

Nicht von ungefähr ist Freud auf die beschriebene Technik gekommen. Er hat sie nicht «erfunden» sondern vielmehr «entdeckt», nachdem er die unerwartete, ja enttäuschende Erfahrung machen musste, dass es keinen direkten Zugang zum Unbewussten gibt, dass auch aus den Träumen das Verborgene nicht direkt ablesbar ist. Im Fall Dora war ihm die Behandlung durch sein intensives Interesse an ihrer Sexualität entglitten.

Nicht mit gezielter Suche also, deren Ergebnis er schon weiß, nicht mit aufdringlichen Interpretationen, nicht kraft seiner ärztlichen Position, sondern durch ein voraussetzungsloses Sicheinlassen auf den Patienten kommt «Analyse» zustande.

Die Stärke der psychoanalytischen Behandlung ist im Grunde noch heute seltsam. Sie liegt in der analytischen Haltung, nicht zuviel zu wollen, sich immer wieder zu befreien vom Wollen und Wissen, um sich immer wieder und in jeder Sitzung neu auf den Patienten einlassen zu können. Zwei Unbewusste, die an einer Kommunikation teilhaben, sollten dies möglichst frei und ungestört tun, der Analysand in freier Assoziation, die Analytikerin mit freischwebender Aufmerksamkeit.

Kommen wir nun zur erwähnten psychoanalytischen «Purifizierung», die von PsychoanalytikerInnen gefordert wird, damit sie von ihren Eigenkomplexen Kenntnis haben, die sie bei der Arbeit stören könnten. 
Mit Purifizierung ist eigentlich eine gründliche Reinigung gemeint. Bei der psychoanalytischen Purifizierung handelt es sich jedoch um einen Reinigungsvorgang, bei dem das Ziel ist, die eigenen neurotischen Anteile als solche mit ihrem Gefahrenpotential für die psychoanalytische Arbeit mit den PatientInnen zu erkennen. Ich finde einmal mehr bemerkenswert, wie Freud mit nüchternem Realitätssinn keine Unfehlbarkeit vorschwebt, auch wenn er sie mit dem Wort «Purifizierung» in den Raum stellt. Der Widerspruch bleibt unaufgelöst.

Was ich bei früheren Lektüren dieses Artikels immer überlesen habe, fällt mir heute auf: die Erwähnung der wünschenswerten Schonung des Affektlebens des behandelnden Analytikers. Freud macht sich offenbar keine Illusionen darüber, auch wenn er es nicht weiter ausführt, dass das Affektleben in Mitleidenschaft gezogen werden kann bei der psychoanalytischen Tätigkeit. Er weiß um die Wirksamkeit, Ansteckungskraft und Verführung des fremden Unbewussten. Die Interaktion in der analytischen Situation, die darin besteht, sich dem Eigenen in der Begegnung mit dem Fremden auszusetzen.

Halten wir uns kurz vor Augen, dass Freud die Ratschläge im Jahr 1912 schrieb, zu einer Zeit, da er auf die Gegenübertragung aufmerksam geworden war, unter anderem durch die Verführungsgeschichte von C.G. Jung und Sabina Spielrein. Freud hatte dem Freund geschrieben: «Sie bitte ich, jetzt nicht zu stark in die Zerknirschung zu gehen. Ich selbst bin nicht ganz so hereingefallen, aber ich war einige Male sehr nahe daran!» ( I. Grosz-Ganzoni 1998 [1993]: 66).

In diesem Zusammenhang versprach Freud 1909 Ernest Jones ein kleines Merkbuch mit Anleitungen und Regeln für die Technik, das nur an die ihm nächststehenden Analytiker hätte verteilt werden dürfen; was jedoch nie erschienen ist.

Die «Ratschläge», die er drei Jahre später veröffentlichte, dienten vermutlich auch der Zügelung der gefährlichen Gegenübertragungsgefühle.

Bis heute sind Freuds Arbeiten (1911-1915) zur Technik der Psychoanalyse so etwas wie das Rückgrat für alle psychoanalytischen Behandlungen. Sie mögen diskutiert, verschrien, als veraltet angesehen oder als Kanon betrachtet werden, sie sind die Referenzpunkte geblieben, auf die zurückgegriffen werden muss in der Auseinandersetzung über technische Fragen in der Psychoanalyse, auch in der Frage zum Unterschied zwischen hochfrequenter Analyse und niederfrequenter psychoanalytischer Psychotherapie.

Währenddem Freud im erwähnten Artikel behauptet, die Technik sei eine leichte, heißt es 20 Jahre später, in seiner 1932 geschriebenen Arbeit «Neue Folge der Vorlesungen zur Einführung in die Psychoanalyse», im Kapitel «Aufklärungen, 
Anwendungen, Orientierungen»: «Die psychoanalytische Tätigkeit ist schwierig und anspruchsvoll, sie lässt sich nicht gut handhaben wie eine Brille, die man beim Lesen aufsetzt und fürs Spazierengehen ablegt. In der Regel hat die Psychoanalyse den Arzt entweder ganz oder gar nicht» (Freud 1933a: 581).

Eine radikale Bemerkung! Nicht der Arzt hat die Analyse verstanden, erfasst, ist ein Professioneller, sondern es ist gerade umgekehrt: Die Psychoanalyse hat ihn ganz oder gar nicht. Freud fährt fort: «Die Psychotherapeuten, die sich gelegentlich auch der Analyse bedienen, stehen nach meiner Kenntnis nicht auf sicherem analytischen Boden; sie haben nicht die ganze Analyse angenommen, sondern sie verwässert, vielleicht «entgiftet); man kann sie nicht zu den Analytikern zählen» (Freud 1982 [1932]: 581).

1912 stellt er die Purifizierung des Psychoanalytikers auf, 1932 spricht er vom notwendigen Gift der Analyse.

Sind damit die Vorstellungen über das psychoanalytische Subjekt gemeint, welche ein, auch heute noch skandalöses Menschenbild enthalten? Überzeugungen wie diejenige, dass jedes - auch das «normale» - Subjekt unausweichlich geprägt ist von innerer Konflikthaftigkeit und Ambivalenz, von libidinöser wie destruktiver Triebhaftigkeit, gehören ebenso dazu wie diejenige, dass es stets einer unstabilen Balance zwischen Triebhaftem und Narzisstischem ausgesetzt ist, immer mit dem Unbewussten zu rechnen hat und dementsprechend derWirkung des Verdrängten nicht entkommen kann. Ja, es wird - nach Freuds berühmtem Dictum - niemals Herr im eigenen Haus sein können.

Diese Grundvoraussetzungen, welche für alle, nicht nur für unsere Patienten und Patientinnen, immer wieder schwer zu akzeptieren sind, gehören für mich in ihrer Härte zum Giftschrank der Psychoanalyse. Im Weiteren zähle ich die Bedingungen bei der analytischen Arbeit, die sich aus der Methode ergeben, dazu. Otto Fenichel (2001 [1936]) formuliert es so - und zitiert dabei auch Sandor Ferenczi (1919) -: «Die psychoanalytische Technik ist eben eine komplizierte Aufgabe. Ihr Instrument bleibt das Unbewusste des Analytikers, dass das Unbewusste des Analysanden intuitiv erfasst. Ihr Ziel ist, dieses Erfassen aus der Intuition herauszuheben zu naturwissenschaftlicher Klarheit. Die analytische Therapie verlangt vom Arzt «einesteils das freie Spielenlassen der Assoziationen und der Phantasie, das Gewähren lassen des eigenen Unbewussten; (...) andernteils muss der Arzt das von seiner und des Patienten Seite gelieferte Material logisch prüfen und darf sich in seinen Handlungen und Mitteilungen ausschließlich vom Erfolg dieser Denkarbeit leiten lassen»» (Fenichel 2001 [1936]: 35). Fenichel (2001 [1936]: 30): «Das Durchkommen zwischen der Scylla des Redens statt Erlebens, 
und der Charybdis des Unsystematischen, nicht von einer übergeordnete Ziele im Auge habenden Ratio erfassten, dem «Agieren` des Patienten entsprechenden 〈Schwimmens», ist (...) die ständige Hauptaufgabe des Analytikers.»

Sich also im Nicht-Verstandenen zu bewegen, das heißt trotzdem denken, schweigen oder reden zu können, sich der Ungewissheit der Übertragung auszusetzen, dabei unter Umständen eigenes Verschwinden und zum Verstummen gebracht werden auszuhalten, sind Situationen, die zum Alltag der analytischen Arbeit gehören. Im Nachhinein klüger zu sein und dabei dieser Tatsache Sinn zu geben, Zweifel und Misserfolge nicht nur zu reflektieren, sondern auch noch fruchtbar zu machen versuchen, runden die unvollständige Aufzählung notwendiger analytischer Kompetenzen ab. Ich meine, das könnte man durchaus als «giftträchtig» bezeichnen - ungefährlich jedenfalls ist er nicht, der Umgang mit dem eigenen und fremden Irrationalen, dem Subjektiven, der psychischen Realität!

«... und wir verlangen daher, dass er (der Arzt [IG]) seine Tätigkeit mit einer Selbstanalyse beginne, und diese, während er seine Erfahrung an Kranken macht, fortlaufend vertiefe. Wer in einer solchen Selbstanalyse nichts zustande bringt, mag sich die Fähigkeit, Kranke analytisch zu behandeln, ohne weiteres abzusprechen» (Freud 1910d: 108).

2

2008

Das Psychoanalytische Seminar führt seit Beginn - ähnlich wie wohl alle psychoanalytischen Institutionen - eine hochengagierte Diskussion um die bestmögliche Ausbildung zum Psychoanalytiker oder zur Psychoanalytikerin und seit einigen Jahren auch zu psychoanalytischen PsychotherapeutInnen.

In den 68ern, als die Psychoanalyse einen Boom erlebte, zu den Leitwissenschaften gehörte und ihre Deutungsmacht grenzenlos schien, wurde sie für alles und jedes beigezogen, bis hin zur Deutung der Weltpolitik. Dies führte zu ihrer Idealisierung. Die Psychoanalyse wurde kulturprägend. Freuds kulturskeptische Position ging dabei zunehmend verloren.

In der Klinik war die Psychoanalyse aber auch in den 68ern eine unter vielen. $\mathrm{Zu}$ einer sorgfältigen Indikationsstellung gehörte es, den Patienten anstatt einer psychoanalytischen Psychotherapie z.B. eine Verhaltenstherapie zu empfehlen. Die heilende Wirkung der Selbsterkenntnis war trotz boomender Psychoanalyse auch damals nicht jedermanns Sache.

Seitdem hat sich die psychoanalytische Praxis gewaltig verändert. Sie muss der Tatsache Rechnung tragen, dass sich das Arbeitsfeld aufgrund neuer sozialer Realitäten, aber auch auf Grund theoretischerWeiterentwicklungen unaufhaltbar 
erweitert. In der Geschichte der Psychoanalyse hatten neue Erkenntnisse, welche unter anderem Produkte derWechselwirkung von Praxis und Theorie sind, und die Ausdehnung des psychoanalytischen Arbeitsfeldes (Kinderanalyse, Gruppenanalyse, Behandlungen von BorderlinepatientInnen, Psychosebehandlungen) schon immer entsprechende Settingveränderungen zur Folge.

Aber die psychoanalytische Methode ist - wie nie zuvor - unter enormen Druck geraten, muss sich in der Konkurrenz der Therapierichtungen, die durch den Kosten- und Spardruck im Gesundheitswesen angeheizt wird, ihren Platz behaupten. Ihre Effizienz soll sie nun auch weitgehend nach den Kriterien der Medizin beweisen.

Diese großeVeränderung in der Haltung gegenüber der Psychoanalyse bringt konsequenterweise nicht nurVeränderungen in der Praxis mit sich, sondern auch im beruflichen Selbstbild der PsychoanalytikerInnen und PsychotherapeutInnen. Nicht vergessen möchte ich dabei den Druck auf die psychoanalytischen Institutionen, in denen das Denken - das bedeutet für mich immer auch Abwägen, eigene Ambivalenzen erkennen und aushalten, entsprechend Argumentieren, schließlich Entscheidungen treffen und nicht zuletzt Handeln - erschwert sind. Es ist eng geworden, der Druck größer.

$\mathrm{Zu}$ diesem Trend gehört, dass die Verlagerung des klinischen Denkens in Richtung einer Diagnostik geht, die mit der psychoanalytischen Diagnostik, die bei uns vor allem bei der Indikationsstellung ihren Schwerpunkt hat, nicht mehr viel zu tun hat. In den neuen Ordnungssystemen (ICD 10, OPD) werden zwar alle psychischen Störungen mit Detailbesessenheit oder Detailbegeisterung erfasst. Was auf den ersten Blick viel versprechend und hilfreich erscheint, hinterlässt im Widerspruch dazu, bei genauerem psychoanalytischen Hinsehen und Nachdenken, so etwas wie ein Beliebigkeitsgefühl. Weder das spezifische Erleben noch das dementsprechend subjektive Handeln der PatientInnen können damit erfasst werden. Von den eigenen spezifischen Reaktionen auf das Rätselhafte, das bei der Therapeutin ausgelöst wird, ganz zu schweigen. Berthold Rotschild hat sich im Journal Nr. 48 ausführlich damit befasst: «Die Nützlichkeit dieser Systematik ist für die Verbindlichkeit der Diagnosestellung und für die wissenschaftlichen Möglichkeiten einer disziplinierten, nunmehr globalisierten Kommunikation unbestritten. Sie hat nur einen, allerdings ganz entscheidenden Nachteil: dass nämlich die genaue Beschreibung und Klassifizierung eines zutiefst rätselhaften Phänomens, wie dasjenige der seelischen Veränderungen, nicht identisch ist mit der komplexen Tragweite eben dieser Phänomene» (Rothschild 2007, 22 f.). 


\section{Zum Alltag in der psychoanalytischen Praxis}

Jede psychoanalytische Psychotherapie (und von der ist hier die Rede, wenn ich «Psychotherapie» erwähne) hat ein Verstehen auch des anscheinend Unverständlichen zum Ziel. Die psychoanalytische Arbeit fordert von den Therapeuten und Therapeutinnen neben den früher erwähnten Fähigkeiten, auch diejenige, sich für verschiedenste Menschen so interessieren zu können, dass sie bereit sind, sich auf ihre inneren Welten einzulassen und dabei auch verrücktes Erleben zu verstehen versuchen. Ohnmacht, Nichtverstehen, Langeweile und andere unangenehme Gefühle müssen ausgehalten werden. Sowohl Flexibilität zum richtigen Zeitpunkt als auch Hartnäckigkeit sind notwendige Eigenschaften. Unter Umständen braucht es die Fähigkeit, die therapeutische Beziehung lange Zeit alleine weitertragen zu können, nicht aufzugeben. Gleichzeitig sollten die subjektiven emotionalen Reaktionen nicht nur ertragen, sondern vielmehr für die Arbeit fruchtbar gemacht werden.

Während den Stunden besteht die analytische Arbeit ja nicht darin, in Selbstreflexion zu verstummen, um dann eine Deutung zu geben. Es geht auch darum, es zu wagen, sich in die Erzählungen der AnalysandInnen mit eigenen Gedanken einzumischen, den Diskurs mit zu kreieren, bei gleichzeitiger Wahrnehmung und Reflexion. Dieses diskursive Reflektieren, Mitkreieren braucht manchmal Mut, oder anders gesagt, ein Stück Angst muss überwunden werden.

Das Herzklopfen vor gewissen Deutungen - und vorher noch vor gewissen Einfällen - kennen wohl alle psychoanalytisch Arbeitenden.

Nicht nur die klassische, das heißt hochfrequente (drei bis fünf Stunden) Analyse, auch die psychoanalytische Therapie benötigt Regelmäßigkeit, eine gewisse Intensität und dauert ihre Zeit. Ihre Effizienz ist nicht in der Kürze und Schnelligkeit begründet, sondern hat mit Selbsterkenntnis und mit der wiedererlangten Fähigkeit, sich weiter zu entwickeln, zu tun.

In unserem Praxisalltag gibt es neben der hochfrequenten Analyse und niederfrequenten Psychotherapie - immer auf dem Hintergrund psychoanalytischer Theorie - noch andere Verfahren, wie Kriseninterventionen und Beratungen, Elterngespräche bei Kinderanalysen usw. Diese haben andere Ziele und erfordern spezifisches Setting und Technik. Sie seien hier einfach erwähnt.

\section{$4 \quad$ Besonderheiten und Unterschiede im hochfrequenten und niederfrequenten psychoanalytischen Setting}

Im Unterschied zum niederfrequenten, ist es im hochfrequenten analytischen Setting einfacher, dem Ablauf des unbewussten Geschehens zu folgen, in 
die Psycho-Logik zu gelangen und das Übertragungs- und Gegenübertragungsgeschehen zu verstehen. In der Arbeit von Tag zu Tag werden Übertragungsprozesse unter der Einwirkung der regressiven Bewegung, die vor allem von der Lage auf der Couch verstärkt wird, erkennbarer. Das kann so weit gehen, wie es KarlAlbrecht Dreher in seinem Artikel «Niederfrequente Psychoanalyse» beschreibt: «DieWiederholung infantiler Reaktionsmuster und Störungen der Selbstregulation lassen den Analytiker vorübergehend Funktionen für seinen Analysanden übernehmen. Dafür, das heißt zum Schutz des Patienten und um neue Erkenntnisse zu gewinnen, sind Termine in ausreichender zeitlicher Dichte erforderlich» (Dreher 2006: 1084).

Dreher beschreibt unmittelbar einleuchtend, weshalb im niederfrequenten Setting die Analyse der Gegenübertragung besonders anspruchsvoll ist (vgl. Dreher 2006: 1087 ff.).

Die selteneren Termine im niederfrequenten Setting machen jede Stunde in der Tendenz zu etwas Geschlossenem. Das prägt auch die Mikro-Interaktion in der einzelnen Stunde. Gleichzeitig wächst die Notwendigkeit, den Bogen über einen längeren Zeitraum zu spannen. Dieser rhythmische Wechsel an Intensität ist mit einer der Gründe für die Besonderheiten und spezifische Schwierigkeiten im analytischen Prozess.

Nichtwissen und Nichtverstehen mit den entsprechenden Affekten von Beunruhigung und Ängsten, wirken von einem Termin zum andern intensiver und lösen entsprechende Phantasien aus, verstärken unter Umständen die Abwehr oder rufen Widerstände gegen die Therapie hervor. Je weiter die Stunden auseinander liegen, umso besser müssen Patienten in der Lage sein, oder mit der Zeit in die Lage kommen, die Verbindung zum analytischen Prozess und zu sich selbst darin zu halten. In der Regel sind sie dabei auf die Hilfe der Therapeutin angewiesen.

Das Übertragungs-/Gegenübertragungsgeschehen ist in der Regel schwerer zu erfassen und auch schwieriger in die Therapie zu bringen. Das Erkennen und die Einfälle in der Gegenübertragung zu erfassen und fruchtbar zu machen, braucht in der Regel Zeit und inneren Raum, was im niederfrequenten Rahmen weniger zur Verfügung steht. Auf die Arbeit mit der Übertragung und Gegenübertragung zu verzichten, hieße aber, auf eine der entscheidensten Quellen der analytischen Arbeit zu verzichten.

Verstrickungen bleiben eher unreflektiert, auch wenn der Therapeut hilfreich wirkt. Oft besteht die Gefahr, dass Wichtiges wieder verschwindet, v.a. natürlich wenn es unangenehm ist, sei es für PatientInnen oder TherapeutInnen. Konflikte sind also einfacher vermeidbar. Es ist die Therapeutin, die den roten Faden nicht 
aus den Augen verlieren darf, aktiv werden muss. Sie kann sich nicht so selbstverständlich den freien Assoziationen und der freischwebenden Aufmerksamkeit überlassen wie im dichteren Setting.

Wann und wie aktiv zu werden oder - um mit Freud zu sprechen - das Kupfer der Suggestion anzuwenden, ist die schwierige Kunst der niederfrequenten psychoanalytischen Psychotherapie, weil das oben erwähnte diskursive Mitreflektieren, die Möglichkeit, die Dinge sich entwickeln zu lassen, beschränkter sind. Manchmal kommt es mir in meiner Arbeit so vor, als ob ich den PatientInnen von Woche zu Woche ein zu großes volles Paket mitgäbe, aus dem sie dann im Laufe der Tage ihr Eigenes entwickeln und in die nächste Stunde bringen. Die Schwierigkeit besteht darin, die regelmäßig daraus verschwindenden unangenehmen Teile zu entdecken und hervorzuholen. Das geht oft nicht ohne Kränkung der Patienten.

Auch wenn das viel zitierte Kupfer der Suggestion natürlich auch in jeder hochfrequenten Analyse zur Anwendung kommt, hat es dort eine andere Bedeutung und wird es eher als Ausnahme wahrgenommen.

Zum Schluss dieses Kapitels möchte ich noch einmal Karl-Albrecht Dreher zitieren: «Akzentuiert werden in der niederfrequenten Psychoanalyse Gefühle von Alleingelassensein, frühkindliche Erfahrungen ungenügenden Containments, Folgen wechselseitigen Missverstehens. Akzentuiert werden aber auch Betonung von Eigenständigkeit, selbstanalytische Fähigkeiten, Stärkung selbstreparativer Funktionen. Welche Elemente in welchem Masse an Bedeutung gewinnen, hängt von den Fähigkeiten und Grenzen der Dyade in Relation zur Erkrankung des Patienten ab» (Dreher 2006: 1084 f.).

\section{Selbsterfahrung in der Analyse für zukünftige Psychotherapeuten und Psychotherapeutinnen}

Im Unterschied zu einer psychoanalytischen Indikationsstellung, bei der verschiedene zu berücksichtigende Faktoren zu einer hoch- oder niederfrequenten analytischen Behandlung führen, geht es bei der psychoanalytischen Selbsterfahrung für den zukünftigen Psychotherapeuten oder die Psychoanalytikerin um die Errichtung der grundlegenden Voraussetzung zur Ausübung des Berufs.

Seitdem der Staat und damit auch Politik und Wirtschaft die Hände im Spiel haben bei der Therapie-Schulung, stellen sich die alten, oft diskutierten Fragen in neuer Schärfe .

Unterschiedliche «Therapieausbildungsprodukte» sind auf dem Markt erhältlich. Verschiedene Schulen haben ein hohes Interesse an der Ausbildung von jungen TherapeutInnen. Es mag immer auch ein lukratives Geschäft sein, vor 
allem aber - das möchte ich besonders hervorheben - sind alle therapeutischen Schulen auf Nachwuchs angewiesen! Dies gilt auch für das Psychoanalytische Seminar Zürich.

Ist es realistisch von uns, für die Selbsterfahrung zum Psychotherapeuten eine hochfrequente Analyse zu empfehlen oder sind wir damit heillos realitätsfremd? Reicht es wirklich nicht, eine psychoanalytische Selbsterfahrungs-Therapie zu machen im Rahmen der geforderten 300 Stunden? Nicht alle wollen schließlich nach der anerkannten chartakonformen Psychotherapieausbildung noch PsychoanalytikerIn werden und hochfrequent arbeiten.

Natürlich drängen sich diese Fragen auf, auch wenn man sich nicht mit dem geforderten modernen Effizienzdenken identifiziert.

Eine zusätzliche Zumutung für das PSZ ist, dass für die anerkannte Weiterbildung in Psychotherapie die Selbsterfahrung in Form einer deklarierten «Lehranalyse» gefordert wird, also etwas, das es in unserer Institution so nie gegeben hat! Im Unterschied zu den andern psychoanalytischen Institutionen, hatte die Schweizerische Gesellschaft für Psychoanalyse (SGP) den weisen Entschluss gefällt, dass jede Analyse erst post festum anerkannt werden solle. Das abgespaltene PSZ übernahm dies auf seine Weise natürlich, meines Wissens ohne Diskussion darüber. Die Idee dahinter war einmal und ist es vielleicht noch immer, dass jede Psychoanalyse eine therapeutische sein soll und damit auch die Frage nach der Motivation für den Beruf Gegenstand der Analyse sein muss. Die älteren AnalytikerInnen unter uns werden sich daran erinnern, dass man bei den ersten Kontaktaufnahmen mit dem Unterrichtsauschuss darauf hingewiesen wurde, dass es keine Garantie gäbe, das man den Beruf einmal ausüben könne, ja es wurde empfohlen, den Analytikerberuf zunächst als Zweitberuf anzugehen. Erst Jahre später - ausgelöst durch den Psychoboom - musste man erstaunt (und nicht ohne Neid) zur Kenntnis nehmen, dass es jüngere KollegInnen gab, welche sich sehr schnell ihre Praxis füllen konnten.

Wenn nun jemand eine psychoanalytische Selbsterfahrung machen muss, um PsychotherapeutIn zu werden, kann das von einem analytischen Standpunkt der möglichst voraussetzungslosen Selbsterkenntnis nur unter derVoraussetzung stattfinden, dass gerade auch die Wahl des Berufes zum Gegenstand der Analyse gemacht wird. Keine Garantie auch heute. Dies aber widerspricht der erwarteten Berufsplanung in der heutigen Zeit. Ich weiß keine Lösung für diesen Widerstreit.

Aber ich hoffe, mit aller Deutlichkeit aufgezeigt zu haben, dass auch für alle zukünftigen PsychotherapeutInnen die eigene psychoanalytische Purifizierung im 
Freudschen Sinn in Form einer hochfrequenten Analyse mehr als empfehlenswert, nämlich meines Erachtens erforderlich und der gesamte Umfang psychoanalytischer Fähigkeiten notwendig ist, um unter den spezifischen, zum Teil erschwerten Bedingungen der niederfrequenten Therapie Erfolge zu haben, die jenen der hochfrequenten Analyse ähnlich sein können.

Ich weiß nicht, ob es notwendig ist, auch «angefressen» zu sein von der Psychoanalyse, etwas, was nicht einzufordern ist. (Im Gegenteil, wir haben ja gelernt, dass einen die Psychoanalyse entweder ganz oder gar nicht hat ). Sicher ist, dass der Beruf des Psychotherapeuten wie der Analytikerin eng mit der Person der Therapeutin/des Analytikers verbunden ist, da es dabei, wie oben beschrieben, nicht nur um Experten-Wissen, sondern um unterschiedlichste seelische Kapazitäten geht.

\section{Literatur}

Dreher, Karl-Albrecht (2006): Niederfrequente Psychoanalyse. In: Psyche 11, 1077-1104.

Fenichel, Otto (2001 [1936]): Probleme der psychoanalytischen Technik. Giessen: Psychosozial Verlag, 2001.

Ferenczi, Sándor et al. (1919): Zur Psychoanalyse der Kriegsneurosen. Wien: Internationaler Psychoanalytischer Verlag.

Freud, Sigmund (1910d): Die zukünftigen Chancen der psychoanalytischen Therapie, GWVIII. Frankfurt: Fischer Verlag.

Freud, Sigmund (1912e): Ratschläge für den Arzt bei der psychoanalytischen Behandlung, GW VIII. Frankfurt: Fischer Verlag.

Freud, Sigmund (19323a): Aufklärungen, Anwendungen, Orientierungen. In: ders., Neue Folge der Vorlesungen zur Einführung in die Psychoanalyse. Studienausgabe I. Frankfurt: Fischer Verlag.

Grosz-Ganzoni, Ita (1998 [1993]): Auf den Spuren der Verführung in der Psychoanalyse. In: Werkblatt40(1), 3-26.

Rothschild, Berthold (2007): Über die Veränderung in Psychiatrie und Psychotherapie. In: Journal für Psychoanalyse 48, 17-27. 